\title{
STUDY OF BTA, HUBBLE, AND SPITZER GRB 021004 DEEP FIELDS
}

\author{
I.V. Sokolov ${ }^{1}$, O.J.A. Bravo Calle ${ }^{2}$ and Yu.V. Baryshev ${ }^{2}$
}

\begin{abstract}
The photometric redshifts were derived, based on photometry data in visual and infrared. Deep fields were observed during gamma-ray bursts investigation with the SAO RAS 6-meter telescope (BTA), Spitzer and Hubble Space Telescope. BTA BVRI images were used, as well as F475W, F606W, F814W of ACS at the Hubble Space Telescope and Spitzer IRAC ch1 $3.6 \mu \mathrm{m}$, ch3 $5.7 \mu \mathrm{m}$. Each BTA BVRI exposure was about one-hour long, reaching objects up to the $\sim 27$ th stellar magnitude. The GRB 021004 field was investigated. A catalog of 874 discovered objects is being made as well as color diagrams and Hubble diagram for these objects.
\end{abstract}

\section{The GRB deep fields}

Deep multi-wavelength surveys are an important tool in studying the formation and evolution of galaxies. Infrared data is particularly useful in these surveys. Several deep fields were observed with the BTA during GRB follow-up campaign. These fields are: GRB 970508 (BVRI), GRB 980703 (BVRI), GRB 990123 (BVRI), GRB 991208 (BVRI), GRB 000926 (BVRI), GRB 021004 (BVRI). The exposures were about 1 hour long. Typical field sizes are $\sim 4^{\prime} \times 4^{\prime}$.

\section{The extraction of objects in GRB 021004 deep fields}

We used visual and IR bands to cover the GRB deep fields. The GRB 021004 field images are presented in Figure 1. These are visual BTA BVRI, Hubble ACS F435W, F606W, F814W, and SPITZER IRAC infrared images. Photometric redshifts of galaxies detected in the field based on BTA/BVRI were measured (Baryshev et al. 2010) using the HyperZ (Bolzonella et al. 2000) software package.

\footnotetext{
${ }^{1}$ Institute of Astronomy RAS, Russia; e-mail: ilia333@land.ru

2 Saint Petersburg State University, Russia
} 

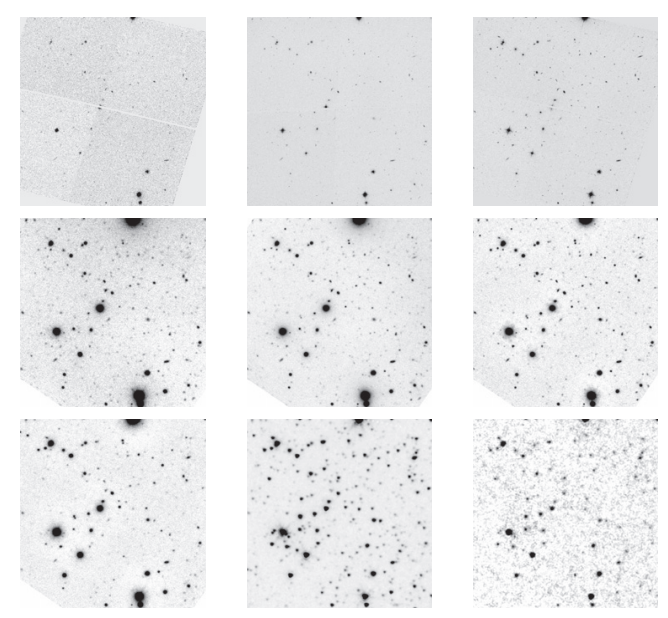

Fig. 1. GRB 021004 field: three upper images are taken with HST, the next four are taken with BTA (the middle row and bottom left), and the last two are taken with the Spitzer Space Telescope. Field sizes are $4^{\prime} \times 4^{\prime}$.

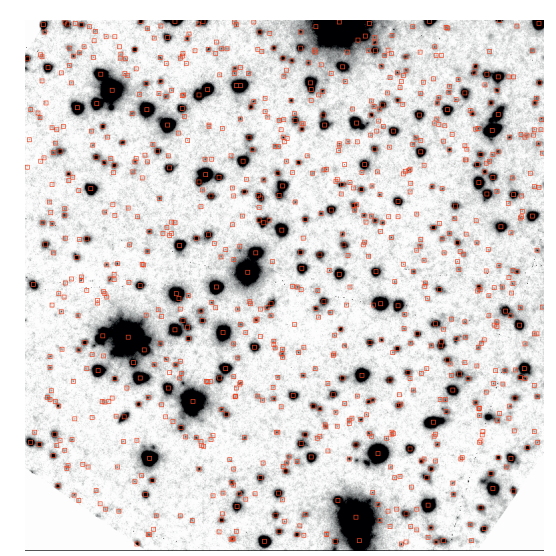

Fig. 2. The stack of all bands, which was used for detection of objects. 874 objects were extracted. The objects are marked with red boxes.

We used the software package SExtractor (Bertin et al. 1996) for the search and photometry of a large number of objects in the field. We used the the dualimage mode, where the stack of all images is used for detection and the photometry is performed on the single images. Used this way, the SExtractor measures the flux in the exact same apertures in all bands. The "STAR CLASS" parameter of the SExtractor package served as a criterion for separating the star-shaped and extended objects. An object is considered star-shaped if its "STAR CLASS" parameter is greater than 0.7 . The $3 \sigma$ excess of intensity over the background was selected as a detection limit, where $\sigma$ stands for the background fluctuations. 

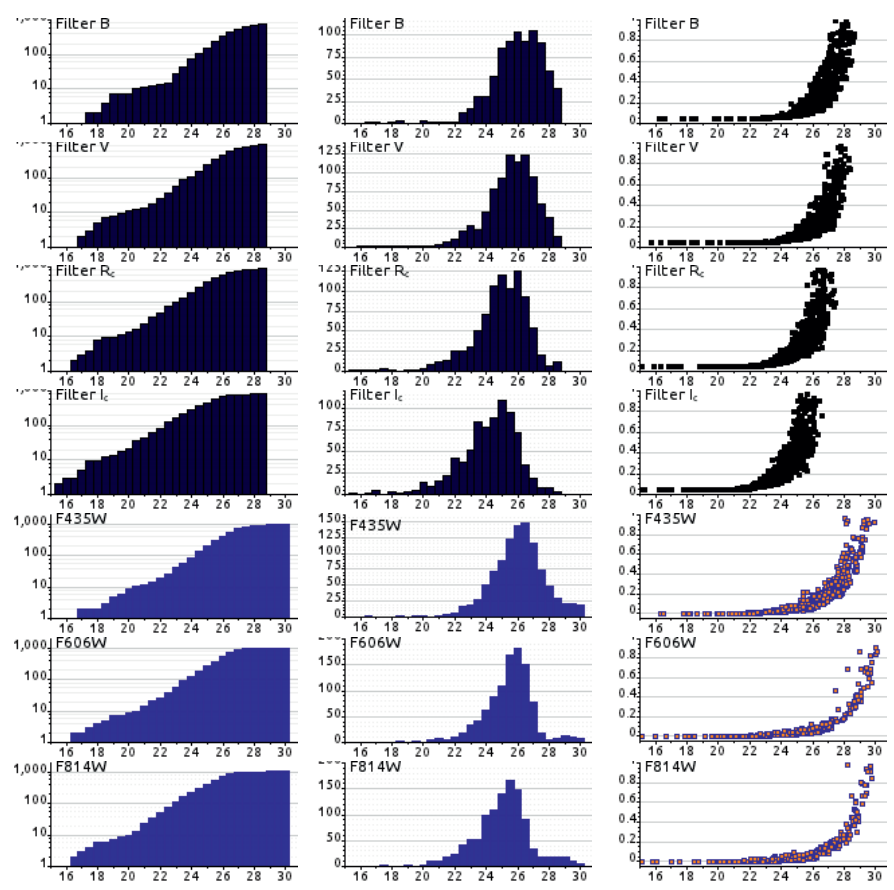

Fig. 3. Magnitude-count, differential magnitude-count and magnitude-error diagrams for 874 objects. Top four rows are for BTA/BVRI, bottom three rows for HST F435W, F606W, F814W. The $\mathrm{x}$-axis is magnitude. The $\mathrm{y}$-axis in first two columns is the number of objects, in the third column - the magnitude error. The diagrams show the depth of the images.

A detected candidate is considered a real object if it occupies at least four adjacent elements of the CCD chip.

\section{Conclusion}

The catalog of 874 detected objects will be available online. It includes the photometry data in each of the nine used bands for each of the detected object. Other GRB deep fields are going to be processed in the similar way.

BTA/BVRI/GRB 021004 redshift estimates (Baryshev et al. 2010) can be considered only as a first approximation. It is supposed to measure photometrical redshifts more precisely with these HST and SPITZER observations (Fig. 1).

\section{References}

Baryshev, Yu.V., Sokolov, I.V., Moskvitin, A.S., et al., 2010, Study of faint galaxies in the field of GRB 021004, Astrophys. Bull., 65, 327

Bertin, E., \& Arnouts, S., A\&AS, 1996, 117, 393

Bolzonella, M., Miralles, J.-M., \& Pelló, R., 2000, A\&A, 363, 476 
